\title{
Comportement hydromécanique des fractures naturelles en milieu granitique
}

Kun SU

Groupement d'intérêt public pour l'étude des Structures souterraines de stockage (GIP-G.3S)

École polytechnique 91128 Palaiseau Cedex

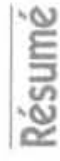

Cette communication présente les résultats des essais hydromécaniques réalisés sur des fractures naturelles d'un granite. Un dispositif spécifique est présenté, permettant d'effectuer dans une cellule triaxiale des essais de cisaillement et de compression sur une fracture colmatée ou non. Sur plusieurs fractures naturelles, les essais mécaniques avec différents chargements sont effectués. Ils sont accompagnés des tests hydrauliques au gaz ou à l'eau. Ainsi, on obtient les propriétés mécaniques et hydrauliques des fractures de même que les paramètres de couplages. Le comportement mécanique des fractures colmatées du granite est plutôt linéaire tant que les fractures sont fermées. La perméabilité hydraulique initiale des fractures colmatées est du même ordre de grandeur que celle du granite sans fracture $\left(10^{-20} \mathrm{~m}^{2}\right)$. Les propriétés hydrauliques changent significativement lorsque la résistance au cisaillement est atteinte. L'ouverture hydraulique à l'eau est de l'ordre de $10 \mu \mathrm{m}$ une fois la fracture rompue par le cisaillement. On constate que la perméabilité à l'eau des fractures est cinq fois moins élevée que celle au gaz.

\section{Hydromechanical behaviour of natural fractures in a granite formation}

This paper is devoted to the exprimental study of hydromechanical coupled behaviour of natural fractures. A new experimental set up is presented allowing to perform shear and compression test on closed or open discontinuities. The are submitted to different mechanical loadings accompanied hydraulic tests using gaz or water at different stress levels. Both mechanical and hydraulic properties of discontinuities as well as coupled parameters are obtained. It is found that closed fractures of granite have a linear mechanical behavior before their failure. The initial hydraulic permeability of these fractures is of the same order as that of granite without fracture $\left(10^{-20} \mathrm{~m}^{2}\right)$. Significant change in permeability beyond shear strenght is observed. Hydraulic aperture to water calculated using a cubic law is about $10 \mu \mathrm{m}$ after failure of natural fractures. Furthemore permeability to water is five times less then that to gaz. 


\section{Introduction}

Les formations cristallines figurent parmi les milieux géologiques étudiés dans nombreux pays pour le stockage de déchets radioactifs. Comme c'est souvent le cas des massifs cristallins, cette formation géologique est caractérisée par des discontinuités essentiellement colmatées à différentes échelles.

Il convient de noter que dans la suite le terme " fracture » est utilisé pour les discontinuités marquées, visibles, avec ou sans remplissage. Ces discontinuités sont étudiées du fait de leur incidence sur l'étanchéité du milieu géologique, car la matrice granitique a une résistance élevée et une très faible perméabilité. Ces propriètés sont-elles susceptibles d'être altérées par suite de sollicitations mécaniques et thermomécaniques? Cette question constitue l'objet de l'étude présentée.

Revue bilbiographique: La rugosité des épontes est une caractéristique importante des fractures. De nombreux auteurs (Gentier et al., 1990; Belem et al., 1993; Hakami et Barton, 1990; Schimittebuhl et al., 1988) ont étudiẻ la morphologie des épontes des fractures. Diverses techniques de caractérisation de la rugosité ont été employées, parmi lesquelles le variogramme et la densité spectrale de puissance constituent les techniques les plus utilisées. Une différence entre les valeurs de la dimension fractale, calculées à partir de ces deux méthodes, a été remarquée par Huang et al, (1991), Belem et al. (1993). La simulation de l'écoulement à partir d'images numériques a été étudiée par plusieurs auteurs (Mourzenko et al., 1995; Amadei et al., 1994; Brown et al., 1987). Mourzenko a réalisé des calculs en 3D afin de modéliser l'écoulement dans une fracture gràce à l'équation de Stokes. L'auteur précise que la différence de débit entre le résultat d'un modèle en 2D et celui en 3D est très grande. Par ailleurs, Didry et Su (1997) ont modélisè les interactions thermo-hydromécaniques d'une fracture isolée dans un milieu continu régi par un comportement thermo-élastique. Cette étude a permis de hiérarchiser l'importance des différents couplages intervenants.

L'étude expérimentale des fractures a également fait l'objet de nombreux travaux scientifiques initiés par Boussinesq (1868). Lomize (1951) (cité par Gale, 1990), Louis (1969) sont parmi les premiers investigateurs qui ont proposé des équations concrètes pour l'écoulement dans une fracture, à partir des données expérimentales. Des termes de frottement, liés à la rugosité des fractures, sont proposés et introduits dans les expressions de la perméabilité. Une revue bibliographie approfondie sur le comportement hydraulique des fractures a été présentée par Gale (1990), L'influence de la contrainte normale au plan de fracture obtenue par plusieurs auteurs a été analysée. L'auteur constate une déviation de la loi cubique sous forte contrainte normale. D'autre part, l'expérience faite par Witherspoon et al. en 1980 a permis de conclure que la linéarité entre le débit et la charge hydraulique reste valable lorsque l'ouverture est supérieure à $4 \mu \mathrm{m}$. Sibai et al. (1997) ont dévéloppé un dispositif permettant de mesurer la pression interstitielle à l'intérieur d'une fracture soumise à des charges hydraulique et mécanique. La distribution non linéaire de la pression des pores dans la fracture a été mise en évidence. La morphologie des fractures joue un rôle essentiel dans la répartition de la pression des pores.
Il est à noter que les études présentées dans les références sont en général réalisées sur des fractures rompues ou préalablement créées par des essais de traction (essai Brésilien, par exemple).

Objet de l'étude: Nos études portent sur le comportement des discontinuités naturelles colmatées à l'état initial. Ces fractures ont été prélevées à partir de sondages dans le granite. Elles présentent une très faible porosité. Un dispositif spécifique a été développé et permet d'effectuer des essais de cisaillement et de compression sur des fractures naturelles. Après une présentation succincte du dispositif, les résultats expérimentaux sont présentés et analysés. Les couplages hydromécaniques et la différence entre la perméabilité au gaz et à l'eau mise en évidence permettent de caractériser le comportement hydromécanique des fractures.

\section{2}

\section{Dispositif expérimental}

L'étude expérimentale du comportement mécanique de la fracture se fait, en général, à partir d'une machine de cisaillement. Ce type d'essais peut être réalisé sur des discontinuités orientées dans la direction de l'application du cisaillement. Or, dans notre cas, les discontinuités sont verticales ou sub-verticales et on dispose des échantillons cylindriques issus des sondages dont le diamètre n'excède pas $90 \mathrm{~mm}$. C'est pourquoi, nous avons été amenés à concevoir un autre type d'essai de cisaillement compatible avec les échantillons disponibles et l'orientation des fractures. Cet essaj devait en plus permettre d'imposer des sollicitations thermo-hydromécaniques, ce qui n'est pas en principe le cas des essais de cisaillement traditionnels. Nous avons développé une nouvelle méthode pour procéder à l'essai de cisaillement dans une cellule triaxiale équipée de circuits hydrauliques et de température, en y apportant certaines modifications. La figure 1 illustre le principe du dispositif mis au point.

L'éprouvette se présente sous une forme cylindrique. Une fracture naturelle (ou créée préalablement à l'essai) traverse l'axe de l'éprouvette. On ôte $5 \mathrm{~mm}$ à l'une des deux extrémités de chaque moitié de l'éprouvette (Fig. 1). De cette façon, on peut admettre un mouvement relatif entre les deux épontes des fractures. L'éprouvette est gainée et ensuite placée dans une cellule triaxiale (Fig. 2). Le rapprochement des talons, supérieur et inférieur, crée une contrainte de cisaille-

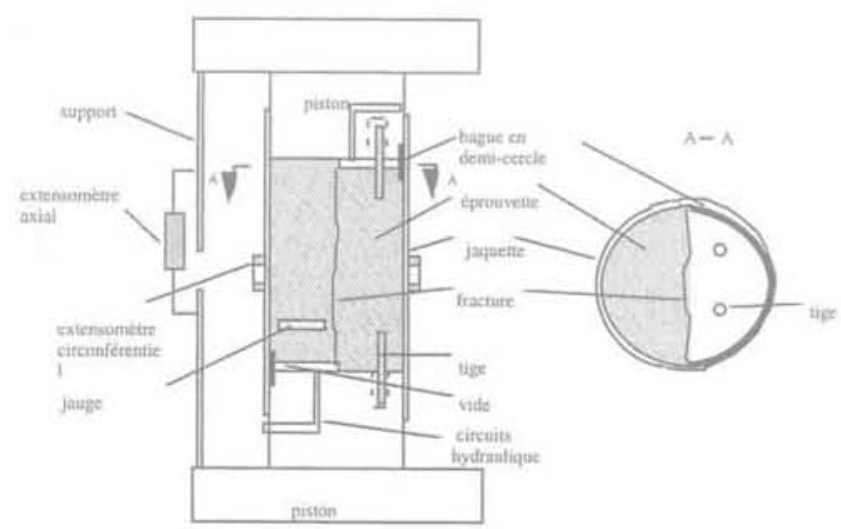

FIG. 1 Dispositif de cisaillement à l'intérieur d'une cellule triaxiale. Shear test device placed inside a triaxial cell. 
ment sur le plan de la fracture. Comme la force est décentrée, un mouvement de basculement a tendance à se produire, rendant l'éprouvette instable. Pour garantir la stabilité de l'éprouvette, quatre tiges en acier sont fixées sur une des deux moitiés de l'éprouvette, dans des trous de $8 \mathrm{~mm}$ de diamètre. Ces tiges sont vissées sur les talons. En conséquence, le mouvement horizontal de la demi-éprouvette hébergeant les tiges est bloqué, mais l'autre moitié est libre de son mouvement horizontal. Sur les deux extrémités, deux bagues en demi-cercle spéciales ont également été utilisées afin d'empêcher la pénétration du fluide de confinement dans les extrémités de l'éprouvette.

La méthode de cisaillement adoptée permet d'utiliser les possibilités offertes par la cellule triaxiale à haute pression, à savoir: circuits hycrauliques, dispositifs de mise en température (chauffage de la cellule), pression de confinement (c'est-à-dire la contrainte normale à la fracture), etc.

La cellule (Fig. 2) a été conue et mise au point par G.3S, et installée sur une presse MTS de $1500 \mathrm{kN}$ de grande rigidité $\left(10^{\circ} \mathrm{N} / \mathrm{m}\right)$. Un système d'asservissement hydraulique précis de la pression de confinement a été conçu également par G.3S pour cette cellule. Il permet d'atteindre une pression de $100 \mathrm{MPa}$.

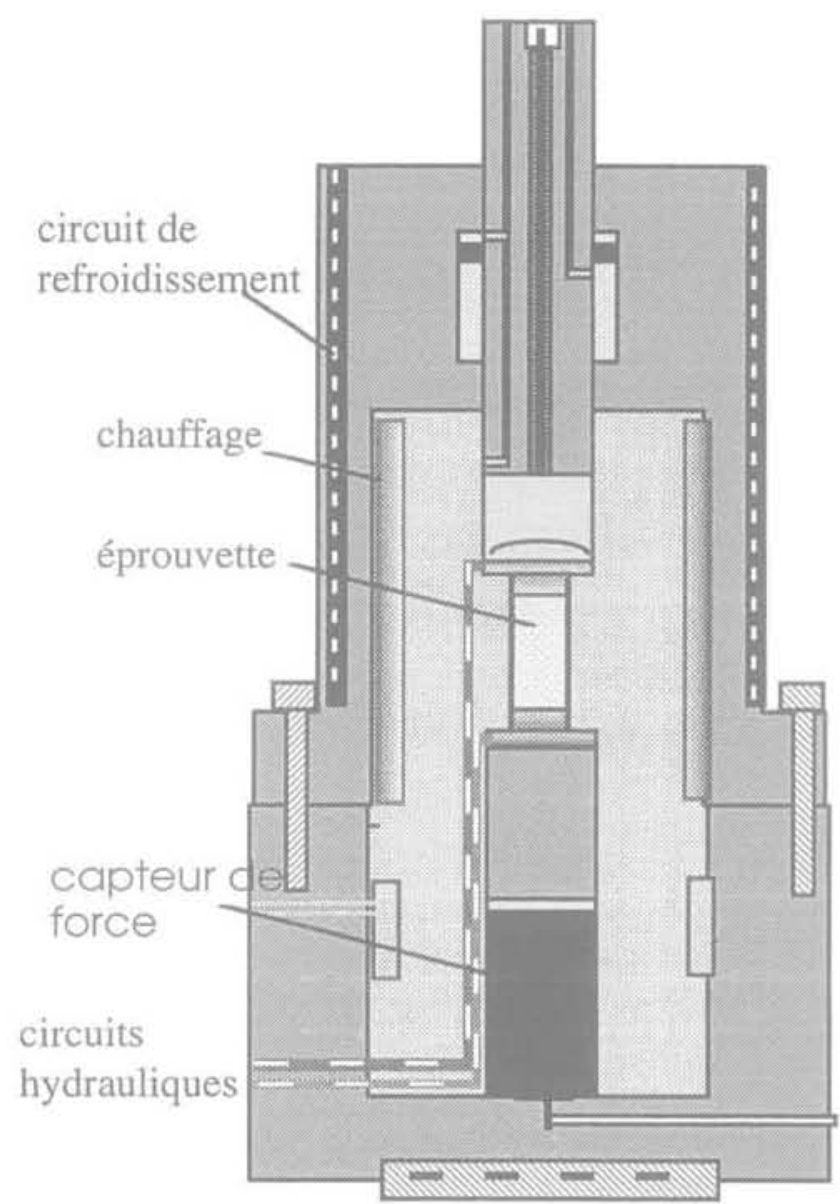

ศG. Q Schéma simplifié de la cellule triaxiale. Triaxial cell scheme.

La force axiale peut être complètement indépendante de la pression de confinement. Ainsi, on peut exercer une contrainte normale au plan de fracture sans influer sur la contrainte de cisaillement.
Les mesures des déformations sont réalisées par des extensomètres MTS placés volontairement sur l'éprouvette à l'intérieur de la cellule. L'extensomètre axial est fixé sur un support permettant de mesurer le dẻplacement relatif des épontes, tandis que l'extensomètre circonférentiel entoure le périmètre de l'éprouvette par une chainette, et mesure la variation circonférentielle de l'éprouvette. Les extensomètres ont une résolution meilleure que $10^{-6}$ en déformation et fonctionnent, sans dérive sous pression élevée (140 MPa) et sous température $\left(200^{\circ} \mathrm{C}\right)$.

Par ailleurs, une jauge de déformation a été collée sur la partie saine de l'éprouvette, permettant ainsi de mesurer la déformation de la matrice du granite. On en déduit la déformation circonférentielle, et on obtient donc la déformation de la fracture.

Il convient de rappeler que les extensomètres doivent être installés sur la gaine, isolant l'échantillon de thuile de confinement. La mesure des déformations locales est perturbée lorsque la pression de confinement varie. Par ailleurs, une jaquette très fine se perce souvent au cours d'un essai qui demande une longue préparation.

Ainsi, nous sommes contraints d'utiliser une jaquette relativement épaisse de 1,6 mm (en Viton). Pour corriger l'effet de la compressibilité de la jaquette, nous avons effectué sur une éprouvette en acier des essais de compression isotrope. Avec l'hypothèse (justifiée) du comportement isotrope de l'éprouvette, nous pouvons déduire la déformation de la jaquette en se référant à la déformation axiale. De cette manière, une correction a été apportée à la déformation circonférentielle. La formule adoptée de correction pour la déformation latérale est un polynôme d'ordre 2:

$$
\varepsilon_{\text {Int }}=\varepsilon_{\text {lat }}^{\prime}+2,2977.10^{-5} \mathrm{P}-4,9266.10^{-8} \mathrm{P}^{2}
$$

où $\mathrm{P}$ étant la pression de confinement en $\mathrm{MPa}, \varepsilon_{\text {, }}^{\prime}$ étant la déformation circonférentielle mesurée par l'extensomètre, sans tenir compte de l'effet de la pression.

La figue 3 illustre les déformations axiale et latérale (mesurées et corrigées) de l'éprouvette en acier, en fonction de la pression de confinement pendant un essai de compression isotrope. La déformation latérale corrigée pendant la charge a la même pente que celle de la déformation axiale.

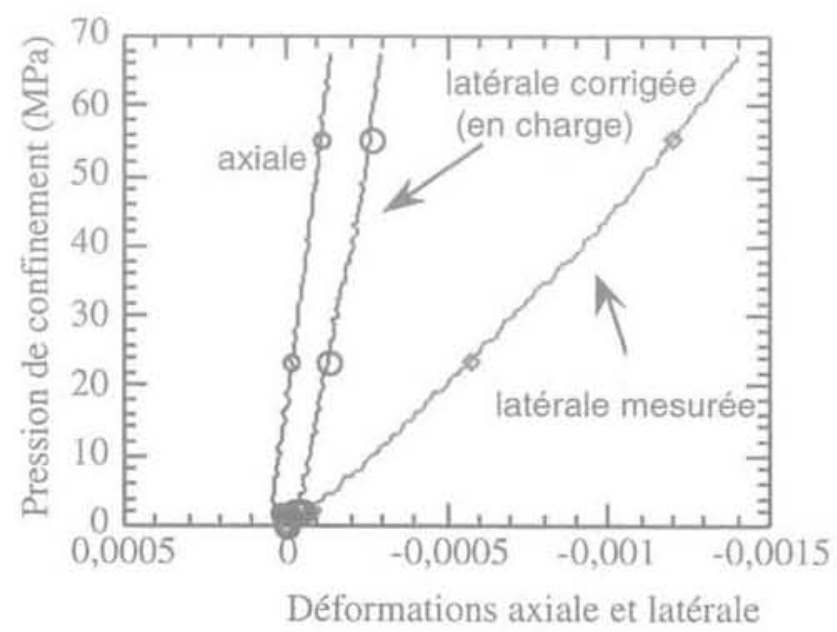

F16.3 Correction de l'influence de la pression de confinement sur la mesure de la déformation latérale.

Correction of the jacket effect for the lateral strain measurement. 


\section{Procédure des essais}

Le montage de l'essai, nous l'avons déjà dit, est relativement complexe et demande un temps de préparation relativement long. Pour les fractures naturelles, l'échantillon se fait rare. Il est donc nécessaire d'adopter une méthodologie adaptée permettant de tirer un maximum d'informations d'un nombre limité d'essais.

Nous avons mis au point une méthodologie constituée par les étapes de chargement suivantes:

a) Étude de sollicitations normales au plan de fracture et de la perméabilité initiale

Cette étape consiste à soumettre l'éprouvette à différentes contraintes normales, en maintenant constante la contrainte de cisaillement nulle. On effectue la mesure de la perméabilité à différents niveaux de la contrainte normale. Dans le cas où la perméabilité est très faible, la seule méthode opérationnelle permettant de mesurer la perméabilité de l'éprouvette dans un temps relativement court est une méthode en régime transitoire.

La courbe $\alpha$ contrainte normale-variation de l'ouverture n de la fracture renseigne sur le comportement mécanique dans le sens normal à la fracture. On peut déterminer ainsi la raideur normale. A priori, nous ne pouvons pas exercer une contrainte normale au-delà de $20 \mathrm{MPa}$, car les deux demi-cercles des bagues antiextrusion ont une résistance mécanique limitée et sont soumis à une traction. L'épaisseur de la bague est aussi limitée, pour des raisons liées au montage de la jaquette.

b) Étude du comportement au cisaillement d'une fracture et de la variation de la perméabilité au gaz

Après un cycle de charge-décharge de la contrainte normale, une valeur de contrainte normale est imposée à l'éprouvette. Ensuite, nous imposons aux épontes de la fracture une vitesse de déplacement relatif. A plusieurs niveaux de contrainte de cisaillement, nous mesurons la perméabilité. Une décharge est programmée avant d'atteindre la limite de rupture au cisaillement. Une fois la résistance au cisaillement atteinte, on arrête l'application du cisaillement. Dans ce cas, le débit est suffisant et sa mesure s'avère possible. Alors, on impose deux pressions différentes aux deux extrémités de l'éprouvette et on mesure le débit du gaz par l'intermédiaire d'un débitmètre. Ainsi, on mesure la perméabilité au gaz de la fracture. Cette perméabilité est comparée aux perméabilités à l'eau mesurée dans la suite de l'essai.

Le comportement au cisaillement de fracture peut aussi être déterminé par ce trajet de charge.

c) Mesure de la perméabilité à l'eau

Une fois la fracture ouverte par cisaillement, on mesure la perméabilité à l'eau sous différentes pressions d'injection ou de pressions de confinement. On s'intéresse, à ce stade, aux propriétés hydrauliques d'une fracture naturelle ouverte par chargement mécanique.

\section{Analyse des résultats}

Trois éprouvettes avec des fractures naturelles plus ou moins parallèles à l'axe de l'échantillon ont été préparées. Rappelons qu'il est difficile de trouver des fractures naturelles parfaitement parallèles à l'axe de
TABLEAUT" Caractéristiques géométriques des fractures naturelles étudiées. Geometric data of the studied natural fractures.

\begin{tabular}{|c|c|c}
\hline$N^{\circ}$ de l'éprouvette & $\begin{array}{c}\text { Epaisseur de } \\
\text { remplissage (mm) }\end{array}$ & $\begin{array}{c}\text { Angle d'inclinaison } \\
(\Omega)\end{array}$ \\
\hline A & 2 & 68 \\
\hline B & 3 & 70 \\
\hline C & 1 & 68 \\
\hline
\end{tabular}

l'éprouvette. Des corrections sont donc nécessaires pour étudier le comportement de la fracture perpendiculaire à ce plan. Le tableau I présente les caractéristiques géométriques des fractures étudiées.

\section{1}

\section{Comportement hydro-mécanique à la compression normale}

La figure 4 illustre la courbe de pression de confinement-déformation latérale corrigée de l'éprouvette B (matrice rocheuse + fracture). La déformation est mesurée, comme nous l'avons déjà précisé, à l'aide d'un extensomètre circonférentiel MTS. La pente de la courbe correspond au rapport E/(1-v), E et v étant le module d'Young et le coefficient de Poisson respectivement. Pour cette éprouvette, on obtient une valeur de $38 \mathrm{GPa}$ pour la pente.

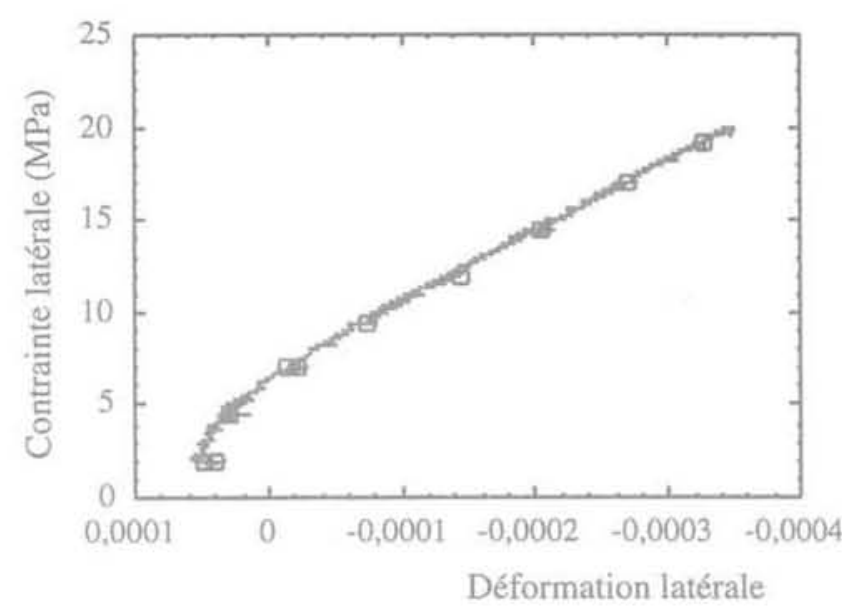

FG.4 Déformation latérale de l'éprouvette (roche + fracture).

Lateral strain of the sample.

Sur la figure 5, est reportée la déformation de la matice rocheuse en fonction de la pression de confinement. Elle est mesurée par une jauge de déformation. La pente de la courbe contrainte-déformation est de $100 \mathrm{GPa}$.

La différence entre les valeurs des modules mesurés s'explique par la présence de la fracture qui a un faible module de compressibilité. Cette différence permet de déduire la variation de l'ouverture de la fracture en fonction de la pression de confinement. Celle-ci est tracée sur la figure 6.

On constate que la fracture naturelle a un comportement linéaire dans le sens perpendiculaire à son plan jusqu'à $20 \mathrm{MPa}$ de contrainte normale. La non-linéarité 


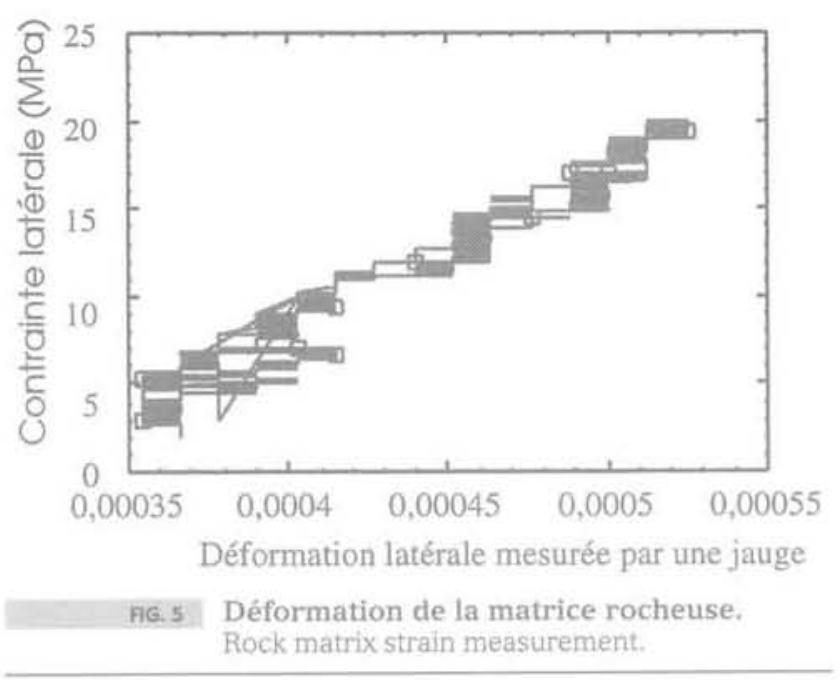

observée au début du chargement est liée à l'effet de la jaquette, et non à la propriété mécanique de la discontinuité. La raideur normale $\left(\mathrm{K}_{\mathrm{n}}\right)$ de cette fracture naturelle est de $633 \mathrm{GPa} / \mathrm{m}$.

II s'agit d'une valeur relativement élevée, qui correspond bien à une fracture colmatée. Comme le comportement est linéaire, en connaissant l'épaisseur de la fracture, on peut exprimer simplement le module d'élasticité de cette fracture par la relation ci-dessous :

$$
\mathrm{E}=\mathrm{K}_{\mathrm{n}} \times \mathrm{e}
$$

avec $: e=$ épaisseur de la fracture, $(e=3 \mathrm{~mm}$ pour cette fracture),

On obtient ainsi : $\mathrm{E}=1,9 \mathrm{GPa}$. Le module de remplissage est environ 50 fois moins élevé que celui de la matrice rocheuse.

Les autres éprouvettes testées manifestent un comportement similaire et les modules de remplissage sont tous de l'ordre de $2 \mathrm{GPa}$.

Dans la littérature, on trouve souvent une expression non linéaire du comportement normal des fractures ou des joints (Goodman, 1976; Zhao, 1992), tandis que nous avons trouvé un comportement linéaire.

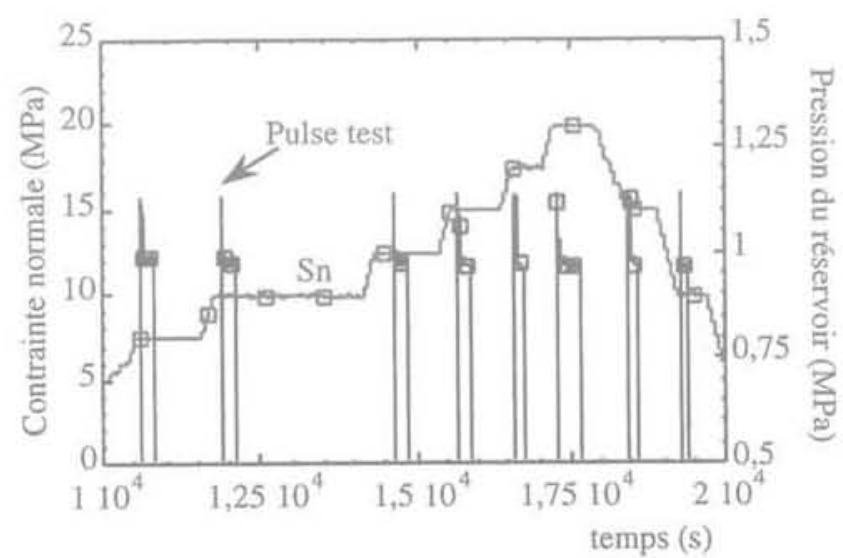

FGG.7 Pression du réservoir en fonction du temps pour différentes contraintes normales (pas de contrainte de cisaillement).

Pressure in the reservoir as a function of time for different values of normal stress (without shear stress).

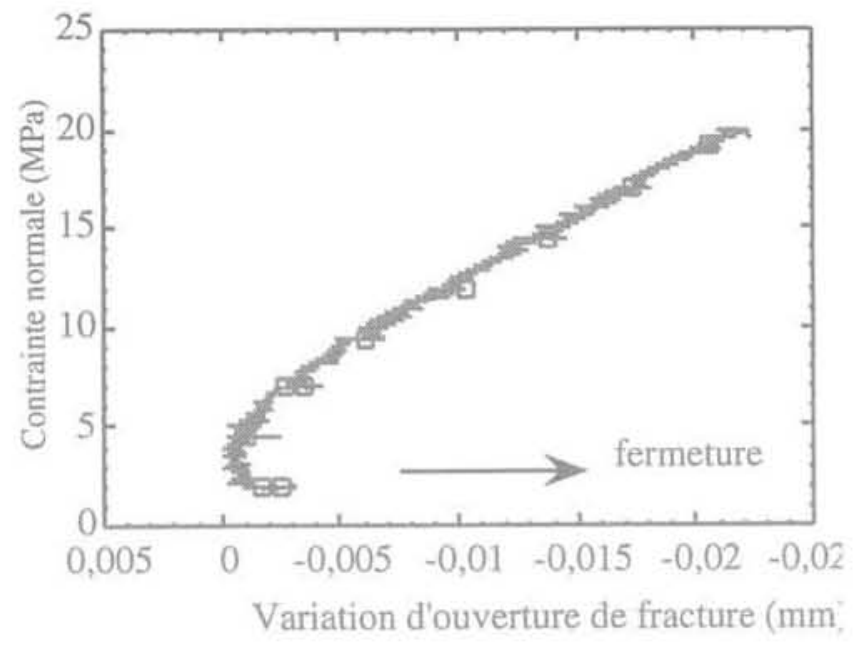

FiG. 6 Variation de l'ouverture de fracture en fonction de la contrainte normale. Variation of fracture opening as a function of normal stress.

La différence peut être due au fait que, dans notre cas, à la différence des autres, il s'agit de fractures naturelles bien colmatées. Le matériau de remplissage manifeste un comportement élastique linéaire en compression. Par conséquent, le comportement de la fracture est linéaire dans la direction normale à son plan.

La perméabilité initiale de la fracture étant faible, la mesure du débit à ce stade s'avère impossible. Ainsi, nous avons été amenés à utiliser la méthode dite du Pulse test. Il s'agit de soumettre l'une des extrémités de l'éprouvette à une pression de gaz contenu dans un réservoir à volume constant. Au fur et à mesure de la pénétration du gaz dans l'éprouvette, la pression du réservoir diminue. La courbe de décroissance de la pression dans le réservoir permet de déterminer la perméabilité. Le détail de cette méthode a été présenté par Su en 1997.

Les figures 7 et 8 illustrent l'évolution de la pression du réservoir en fonction du temps. Comme le montre la figure 7, les essais sont effectués à différents niveaux de

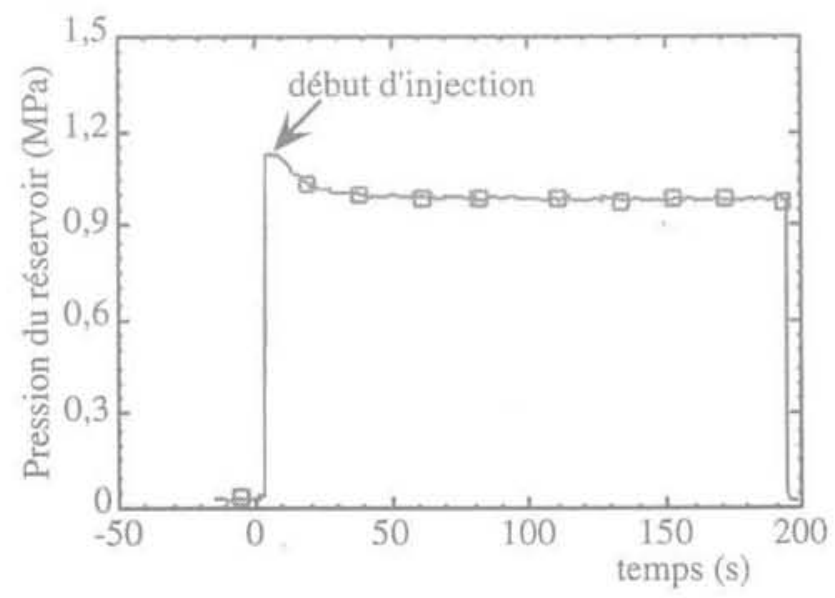

FO. 8 Pression du réservoir en fonction du temps pour une contrainte normale au plan de fracture de $20 \mathrm{MPa}$ (pas de contrainte de cisaillement).

Pressure in the reservoir as a function of time for a normal stress of $20 \mathrm{MPa}$ (without shear stress). 
contrainte normale. La pression du réservoir (connecté au talon inférieur) reste pratiquement constante une fois que le gaz est injecté.

La légère décroissance de la pression en fonction du temps (Fig. 8) signifie que l'éprouvette (fracture + matrice rocheuse) n'est pas strictement imperméable. La perméabilité globale est de l'ordre de $10^{-20} \mathrm{~m}^{2}$ si l'on compare la courbe avec les abaques numériques établis par Su en 1997. Cette valeur est proche de celle de la perméabilité du granite sans fracture. On peut ainsi conclure que la fracture naturelle colmatée a une perméabilité aussi faible que la matrice sans discontinuité.

\section{2}

\section{Comportement mécanique des fractures naturelles au cisaillement}

Les essais de cisaillement sont effectués avec différentes valeurs de contrainte normale maintenue constante au cours d'un essai. On impose une vitesse de déplacement relatif des épontes de fracture à l'aide d'un extensomètre axial. L'ouverture de la fracture est mesurée avec l'extensomètre circonférentiel. Comme la pression de confinement est constante pendant l'essai de cisaillement, la mesure circonférentielle n'est pas affectée par la pression.

La figure 9 présente la courbe «contrainte de cisaillement-déplacement relatif des épontes de fracture » pendant un essai de cisaillement sur l'éprouvette $\mathrm{A}$, la pression de confinement étant de $5 \mathrm{MPa}$. On constate que la discontinuité colmatée a un comportement linéaire avant sa rupture (pic au cisaillement). La raideur de cisaillement de cette fracture est de $\mathrm{K}_{1}$ $=347 \mathrm{GPa} / \mathrm{m}$

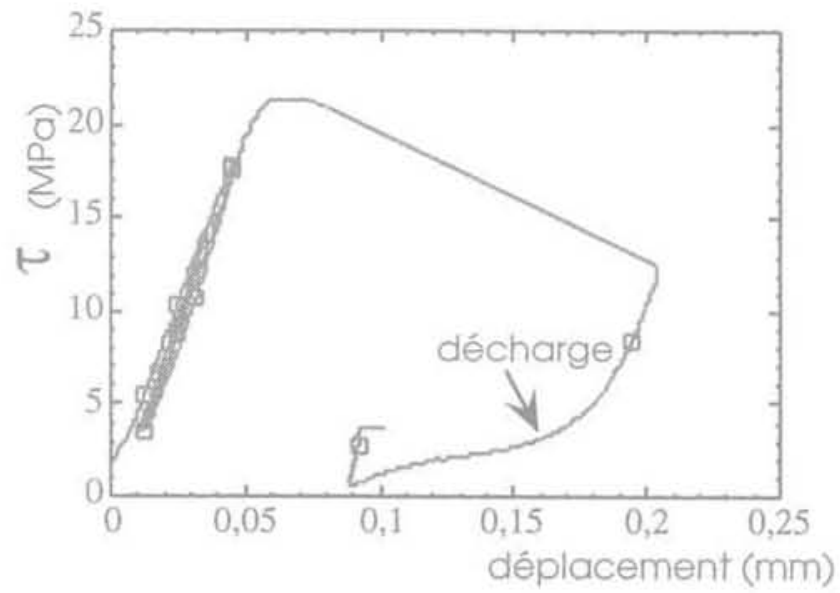

FG. 9 Contrainte de cisaillement en fonction du déplacement relatif des épontes de la fracture.

Shear stress versus relative displacement of fracture walls.

Sur la figure 10, est reportée l'évolution de l'ouverture de la fracture pendant un essai de cisaillement. Comme la pression de confinement est constante, toute variation de déplacement circonférentiel est attribuée à la variation d'ouverture de la fracture. La dilatance est très significative. On définit la pente de la courbe de dilatance qui donne l'angle de dilatance d: nous avons pour cette fracture: $\mathrm{d}=37^{\circ}$

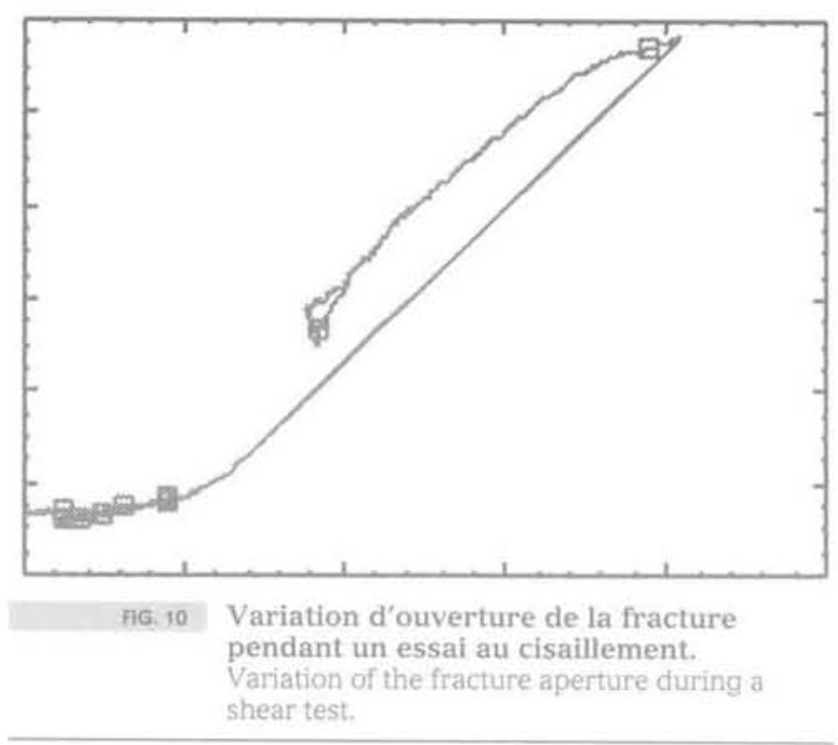

Avant la rupture, la dilatance est relativement faible. La figure 11 montre qu'il y a eu seulement $7 \mu \mathrm{m}$ de dilatance dans la phase (célastique) du cisaillement, soit un angle de $8^{\circ}$.

La transition entre cette faible dilatance $\left(8^{\circ}\right)$ de la phase (célastique » et la dilatance associée au comportement post-rupture se fait au moment de la rupture (Fig. 11). Il est évident que l'angle de dilatance devrait tendre vers une valeur nulle quand le déplacement relatif des épontes devient grand. II faut signaler que le déplacement relatif des épontes de fracture obtenu pendant nos essais de cisaillement est inférieur à $1 \mathrm{~mm}$, valeur probablement trop faible pour permettre d'atteindre la phase de stabilisation de la dilatance de la fracture. La rugosité de la fracture a aussi un rôle important dans la dilatance.

L'angle de dilatance est un paramètre important intervenant dans le couplage hydromécanique. Il exprime l'ouverture mécanique de fracture due au cisaillement.

Le tableau II résume les résultats des essais de cisaillement sur les trois fractures naturelles étudiées, la raideur de cisaillement n'apparait pas très sensible à l'épaisseur de la fracture.

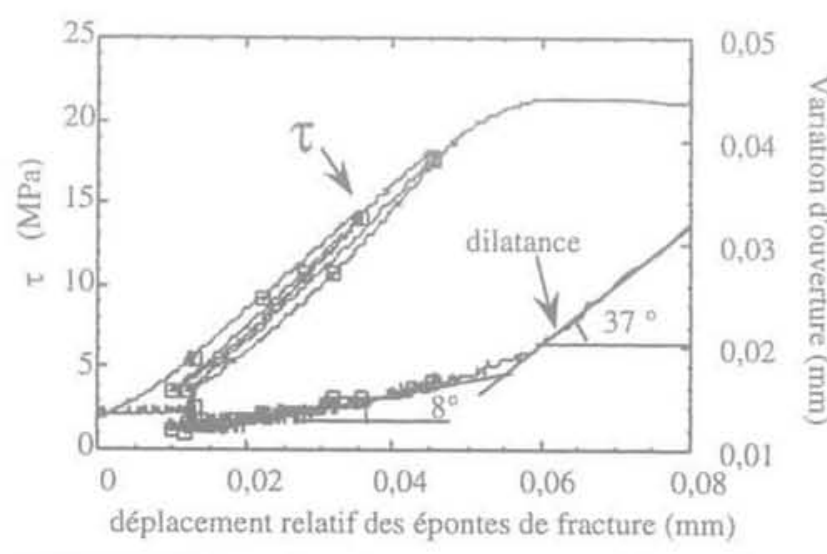

FIG. 11 Dilatance de la fracture pendant la phase élastique au cisaillement.

Dilatance of the fracture in the elastic phase during a shear test. 


\begin{tabular}{c|c|c|c|c|c|c}
$\begin{array}{c}N^{\circ} \\
\text { d'éprouvette }\end{array}$ & $\begin{array}{c}\text { Epaisseur } \\
\text { de la fracture } \\
\text { (mm) }\end{array}$ & $\begin{array}{c}\text { Raideur } \\
\text { tangentielle } \\
(\mathrm{GPa} / \mathrm{m})\end{array}$ & $\begin{array}{c}\text { Contrainte } \\
\text { normale } \\
\text { (MPa) }\end{array}$ & $\begin{array}{c}\text { Résistance au } \\
\text { cisalllement } \\
\text { (MPa) }\end{array}$ & $\begin{array}{c}\text { Angle de dilatance } \\
\text { avant/aprés } \\
\text { la rupture (?) }\end{array}$ & $\begin{array}{c}\text { Déplacement } \\
\text { à la rupture } \\
\text { (mm) }\end{array}$ \\
\hline A & 2 & 347 & 5 & 21,5 & $8 / 37$ & 0,07 \\
\hline B & 3 & 388 & 10 & 38 & $8 / 37$ & 0,13 \\
\hline C & 1 & 555 & 15 & 42 & $7 / 36$ & 0,07 \\
\hline
\end{tabular}

\section{3}

\section{Comportement hydraulique au gaz et à l'eau des fractures naturelles soumises au cisaillement}

Au cours de l'essai de cisaillement, des mesures de perméabilité par la méthode du Pulse test sont effectuées à différents niveaux de cisaillement jusqu'à la rupture. Nous ne constatons aucune variation significative de la conductivité hydraulique de la fracture avant la rupture. Pourtant nous avons observé, grâce à la mesure à l'extensomètre circonférentiel, qu'il existe bien des dilatances de la fracture. Ceci s'explique par le fait que cette dilatance (quelques microns) n'est pas suffisante pour provoquer une percolation significative.

Une fois la fracture rompue, la mesure de la perméabilité au gaz montre que tout à fait naturellement le fluide passe bien à travers la fracture. Sur l'éprouvette $B$, nous avons réalisé des mesures de la perméabilité au gaz suivies par des mesures à l'eau. Le gaz utilisé était de l'azote. Nous avons mesuré le débit du gaz en contrôlant la différence des pressions entre les talons supérieur et inférieur.

En connaissant le débit et le gradient, il est intéressant de calculer l'ouverture hydraulique de la fracture qui est un paramètre clé caractérisant les propriétés hydrauliques des fractures. Pour déterminer cette ouverture au gaz de fracture, deux hypothèses simplificatrices sont faites, à savoir :

$\mathrm{H}_{1}$ : La géométrie de fracture est assimilée à deux plaques parallèles avec une ouverture uniforme;

$\mathrm{H}_{2}$ : Localement, la vitesse moyenne du gaz est régie par la loi de Poiseuille :

$$
V=\frac{e^{2}}{12 \mu} \frac{d P}{d x}
$$

où : P la pression $(\mathrm{Pa})$;

e l'ouverture hydraulique $(\mathrm{m})$;

$\mu$ la viscosité dynamique ( $\mathrm{Pa} \mathrm{s}$ ).

Alors, l'équation de la conservation de masse en régime stationnaire, conduit à la relation suivante:

$$
\frac{d}{d x}(\rho V)=0
$$

soit:

$$
\frac{d}{d x}\left\{\rho \frac{e 2}{12 \mu} \frac{d P}{d x}\right\}=0
$$

$\rho$ est la masse volumique de l'azote; elle est déterminée par l'équation d'état:

$$
p=\frac{P_{0}}{P_{0}} P
$$

$\rho_{0}$ et $P_{0}$ sont respectivement la masse volumique et la pression de référence, pour l'azote $p_{0}=1,1639 \mathrm{~kg} / \mathrm{m}^{3}$ et $\mathrm{P}_{0}=0,1 \mathrm{MPa}$.

Pour une fracture de longueur $L$ avec deux pressions distinctes $\mathrm{P}_{1}(\mathrm{x}=0)$ et $\mathrm{P}_{2}(\mathrm{x}=\mathrm{L})$ imposées respectivement sur les deux extrémités, la solution de la pression s'écrit :

$$
P=\sqrt{\frac{P_{2}^{2}-P_{1}^{2}}{L} \times+P_{1}^{2}}
$$

Le flux volumique $\left(\mathrm{m}^{3} / \mathrm{s}\right)$ traversant la fracture est égal à :

$$
\varnothing=\frac{\mathrm{e}^{3}}{24 \mu \mathrm{P}_{0}} \frac{\left(\mathrm{P}_{2}^{2}-\mathrm{P}_{1}^{2}\right)}{\mathrm{L}} \mathrm{D}
$$

avec: D largeur de la fracture.

Donc en connaissant le débit, on peut calculer l'ouverture hydraulique e:

$$
\mathrm{e}=\sqrt[3]{\frac{24 \mu \mathrm{P}_{0} L \varnothing}{\left(\mathrm{P}_{2}^{2}-\mathrm{P}_{1}^{2}\right) \mathrm{D}}}
$$

Le tableau III présente les résultats des mesures de perméabilité au gaz dans la phase post-rupture du comportement au cisaillement. On constate que l'ouverture hydraulique au gaz est de l'ordre de $20 \mu \mathrm{m}$ quand la contrainte normale est de $2 \mathrm{MPa}$. Par ailleurs, la variation du gradient de pression et de la pression d'injection ne modifie pas significativement l'ouverture hydraulique. Cela signifie que la relation (7) s'applique, et que les hypothèses prises sont justifiées. L'ouverture mécanique à ce stade est de $300 \mu \mathrm{m}$. Le rapport entre l'ouverture hydraulique et l'ouverture mécanique est de 0,07.

L'étape suivante consiste à mesurer la perméabilité à l'eau au lieu du gaz. La contrainte normale est maintenue à $2 \mathrm{MPa}$. Le débit est contrôlé par la mesure de la masse d'eau évacuée par minute. On admet à nouveau la loi cubique et l'incompressibilité du fluide. Dans ce cas l'ouverture hydraulique à l'eau peut être déduite de la relation suivante:

$$
\mathrm{e}=\sqrt[3]{\frac{12 \mathrm{Q} \mu \mathrm{L}}{\Delta \mathrm{PD}}}
$$


TABLEAU III Résultats des mesures de la perméabilité au gaz.

Results of permeability measurements to gas.

\begin{tabular}{|c|c|c|c|c|c|}
\hline Mesure & $\begin{array}{l}\text { Pression du } \\
\text { confinement } \\
\text { (MPa) }\end{array}$ & $\begin{array}{l}\left(\mathrm{P}_{-}-\mathrm{P}_{1}\right) \\
10^{5} \\
(\mathrm{~Pa})\end{array}$ & $\begin{array}{l}\mathrm{P} \\
10^{\frac{7}{2}} \\
(\mathrm{~Pa})\end{array}$ & $\mathrm{Q}\left(\mathrm{cm}^{3} / \mathrm{s}\right)$ & 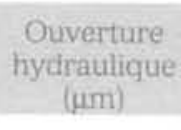 \\
\hline 1 & 2 & 0,34 & 2,3 & 1 & 18 \\
\hline 2 & 2 & 0,17 & 4,8 & 1 & 17 \\
\hline 3 & 2 & 0,195 & 10,7 & 3 & 18 \\
\hline 4 & 2 & 0,22 & 13,4 & 4,17 & 18 \\
\hline
\end{tabular}

où: $Q$ est le débit volumique, $\mathrm{m}^{3} / \mathrm{s}, \mu$ est la viscosité dynamique de l'eau Pa s.

Le tableau IV résume les résultats des mesures de la perméabilité à l'eau. On constate que l'ouverture hydraulique à l'eau est cinq fois moins importante que celle au gaz, cette dernière est seulement de l'ordire de $4 \mu \mathrm{m}$. Cette différence est probablement liée aux effets suivants : - par cisaillement, le remplissage de la fracture est broyé et devient un matériau poreux. Ce matériau, initialement sec, devient saturé ou partiellement saturé à l'eau sous l'effet d'injection de l'eau. Les particules de remplissage peuvent être transportées par l'eau et boucher plus loin les canaux de circulation;

- la taille des pores créés dans la fracture est extrêmement petite. Par le fait que la taille moléculaire de gaz est inférieure à celle de l'eau, alors certains canaux sont probablement inaccessibles à l'eau, mais accessibles au gaz; - il est possible qu'il existe des réactions chimiques entre l'eau et certains minéraux de remplissage. On constate. après l'essai, que le matériau de remplissage se transforme en une sorte de pâte. Ainsi la circulation de l'eau peut être bloquée, ce qui a été observé sur une éprouvette.

Une analyse diffractométrique aux rayons X sur le matériau de remplissage broyé par le cisaillement a été effectuée. Le résultat montre que le remplissage de la fracture est constitué presque, exclusivement de trois phases cristallines, le quartz, la calcite et un minéral phylliteux, sans doute le chlorite. Des études supplémentaires sont nécessaires pour apprécier l'ampleur des effets chimiques éventuels.

TABLEAUN Résulats des mesures de la perméabilité à l'eau sur l'éprouvette B.

Results of permeability measurement to gas obtained on the sample $B$.

\begin{tabular}{c|c|c|c|c}
\hline Étape & $\begin{array}{c}\text { Contrainte } \\
\text { normale } \\
(\mathrm{MPa})\end{array}$ & $\begin{array}{c}\text { Pression } \\
\text { d'entrée } \\
(\mathrm{MPa})\end{array}$ & $\begin{array}{c}\mathrm{Q} \\
\left(\mathrm{cm}^{2} / \mathrm{s}\right)\end{array}$ & $\begin{array}{c}\text { Ouverture } \\
\text { hydraulique } \\
(\mu \mathrm{m})\end{array}$ \\
\hline 1 & 2 & 1,37 & 0,0047 & 4,3 \\
\hline 2 & 2 & 0,81 & 0,0020 & 3,8 \\
\hline 3 & 2 & 0,51 & 0,00017 & 2,0 \\
\hline 4 & 2 & 0,49 & 0,0013 & 4,0 \\
\hline 5 & 2 & 0,84 & 0,0048 & 5,1 \\
\hline 6 & 2 & 1,44 & 0,0050 & 4,3 \\
\hline 7 & 2 & 1,6 & 0,0123 & 4,1 \\
\hline
\end{tabular}

La variation de la pression interstitielle n'a pas d'influence significative sur l'ouverture hydraulique. Quand la pression d'injection passe de $0,49 \mathrm{MPa}$ à $1,6 \mathrm{MPa}$, le débit augmente proportionnellement, mais l'ouverture hydraulique déduite reste pratiquement la même.

Sur l'éprouvette A, l'ouverture hydraulique à l'eau est de l'ordre $9 \mu \mathrm{m}$. Au début de l'essai hydraulique, la perméabilité de la fracture est nulle. La circulation est bloquée. Il a fallu que l'on modifie la contrainte normale et la pression d'injection pour établir une circulation. On constate que la relation linéaire entre le débit et la pression d'injection est maintenue, pourvu que la contrainte normale soit égale à $2 \mathrm{MPa}$ (Fig.12). L'augmentation de la pression d'injection n'a pas entrainé une ouverture hydraulique de la fracture. Pourtant l'extensomètre circonférentiel enregistre une variation d'ouverture de l'ordre de $20 \mu \mathrm{m}$ pour une variation de la pression d'injection de $1 \mathrm{MPa}$. Cela signifie que cette ouverture mécanique ne s'est pas répercutée sur l'ouverture hydraulique.

Quand la contrainte normale augmente à $3 \mathrm{MPa}$, la relation linéaire «débit-pression d'injection » n'est plus linéaire (Fig. 12). L'augmentation de la pression d'injection entraîne une variation de l'ouverture hydraulique.

Ces différents types du comportement hydraulique observés sur une même fracture montrent combien le couplage hydromécanique dans les fractures naturelles initialement colmatées peut être complexe.

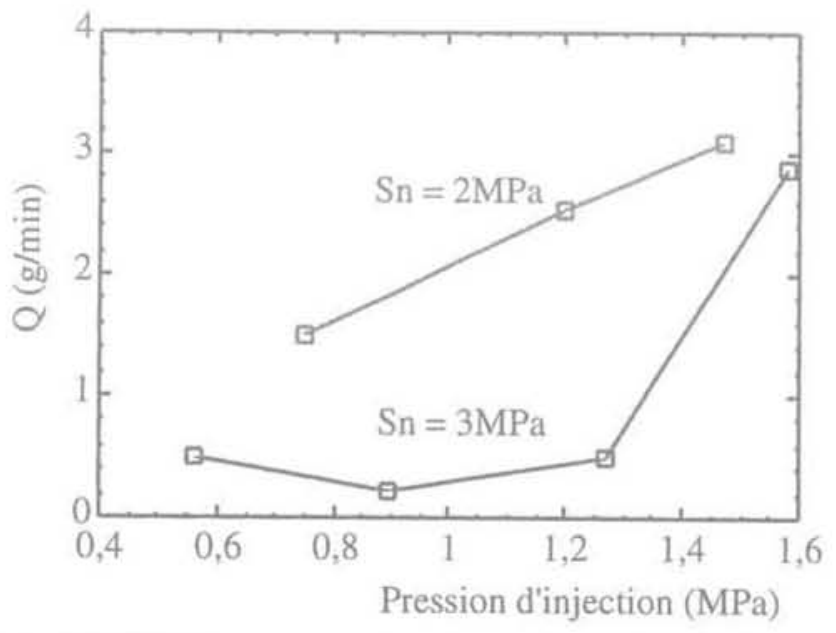

FiG.12 Relation entre le débit massique et la pression d'injection (éprouvette A). Plot of flow rate versus pressure of injection (sample A) 


\section{Conclusion}

Les essais hydromécaniques réalisés sur les disscontinuités colmatées du granite ont apporté des résultats nouveaux et originaux sur le comportement hydraulique et le couplage hydro-mécanique de ce type de discontinuités.

Le comportement mécanique de ces discontinuités colmatées est linéaire avant la rupture au cisaillement. Le module d'élasticité du matériau de remplissage de ces discontinuités est de l'ordre de $2 \mathrm{GPa}$. La raideur normale des fractures colmatées varie linéairement avec l'épaisseur de ces discontinuités. La raideur tangentielle des discontinuités colmatées n'est pas sensible à l'épaisseur et reste voisine de $400 \mathrm{GPa} / \mathrm{m}$. La rupture des discontinuités colmatées intervient lorsque le déplacement relatif des épontes de la fracture atteint 70 à $130 \mu \mathrm{m}$. L'angle de dilatance après la rupture est de l'ordre de $37^{\circ}$. Dans la phase avant la rupture, cet angle est de l'ordre de $8^{\circ}$.
La perméabilité initiale des fractures naturelles étudiées est très faible. Elle est du même ordre de grandeur que celle de la matrice rocheuse. Avant la rupture au cisaillement aucune augmentation significative de la perméabilité n'est constatée.

La rupture des discontinuités entraîne une augmentation significative de l'ouyerture hydraulique. Cette ouverture est de l'ordre de $10 \mu \mathrm{m}$. Le rapport de l'ouverture hydraulique sur l'ouverture mécanique est de l'ordre de $7 \%$. La circulation de l'eau dans une fracture naturelle après rupture est sensiblement liée à la nature du matériau de remplissage et à l'écrasement de ce matériau par le cisaillement. La circulation du fluide peut être bloquée par la présence de petites particules créées par la rupture. L'ouverture hydraulique à l'eau des fractures est cinq fois moins élevée que celle au gaz.

L'analyse de la rugosité des fractures naturelles et de son influence sur le comportement hydraulique des fractures est en cours et sera complétée ultérieurement. Une autre perspective intéressante est l'interprétation poussée des résultats disponibles à partir d'une modélisation hydro-mécanique couplée, si possible à trois dimensions.

\section{REMERCIEMENTS}

Cette recherche a été entreprise avec le soutien et la collaboration de l'Agence nationale pour la gestion des déchets radioactifs (ANDRA). Qu'elle soit assurée de notre gratitude.

\section{Bibliographie}

Amadei B., Illangasekare T.A. - « Mathematical Model for Flow and Solute Transport in Non-homogeneous Rock Fractures i. Int. J. Rock Mech. Min. SCi. \& Geomech. Abstr. vol. 31. n 6, 1994 , p. 719-731.

Belem T., Homand F., Sabbadinl S. a. Influence of Morphology Discontinuities on Shear Behaviour $\mathrm{x}$. Colloque franco-polonais, Nancy, 15-19 nov. 1993, p. $153-162$.

Boussinesq I. - $\propto$ Mẻmoire sur linfluence des frottements dans les mouvement réguliers des fluides p. J. Math. Pure Appl. Ser. 2, 13, 1868, p. $377-424$.

Brown S.R., Scholz C.H. - "Broad Bandwidth Study of the Topography of Natural Rock Surfaces w. Journal of Geophysical Research vol. 90, $\mathrm{n}^{\circ}$ B14, 1985, p. $12575-12582$.

Brown S.R. $-\alpha$ Fluid Flow Through Rock Joints: The Effect of Surface Roughness ग. Journal of Geophysical Research vol. 92, n B2, 1987, p. 1337-1347.

Didry O., Su K - " Assessment of thermohydromechanical interactions for a fractured rock using a finite difference method n. EUROCK'96, Torino, Italy, September 2-5, 1996, p. 1379- 1386. Gale J. - « Hydraulic behaviour of rock joints 1. Proc. Int. Symp. on Rock Joints, Leon Norway, 1990, p. 351-362.

Gentier S., Riss J. - « Quantitative description and modelling of joints morphology n. Proc. Int. Symp, on Rock Joints, Leon Norway, 1990, p. 375-382.

Goodman R.E. - Methods of geological engineering in discontinuous rock. West Publishing Comp., 1976.

Hakami H., Barton N. - $\alpha$ Aperture measurements and flow experiments using transparent replicas i. Proc. Int. Symp. on Rock Joints, Leon Norway, 1990, p. 383-390.

Huang S.L., Speck R.C., Oelfke S.M. «Fractal Characterization and Modeling of Natural Rock Joint. Rock Mechanics as a Multidiscipilinary Science s. 32 nd U.S. Symposium of Rock Mechanics, Oklahoma, 1991, p. 1125-1134.

Louis C. - "A study of groundwater flow in jointed rock and its influence on the stability of rock masses $\mathrm{v}$. Rock mechanics research report, Imperial college, $n^{\circ} 10.1969$

Mourzenko V.V., Thovert J.-F., Adler P.M. - a Permeability of a Single Fracture. Validity of the Reynolds Equation v. . . Phys. II France 5, 1995, p. 465-482.

Okubo G.P. Aki K. $-\alpha$ Fractal Geometric in the San Andreas Fault System 1, Journa of Geophysical Research vol. 92, n B1. 1987 , p. $345-355$.

Schmittbuhl f Vilotte I-P. Roux S - icPercolation through self-affine surfaces $v, J$. Phys. A. Math. Gen. 26, 1993, p. 6115-6133.

Sibai M. Haif Sotoudeh M. Henry J.-P. *. Étude expérimentale du couplage hydromécanique de joints rocheuxn. Revue française de géotechnique, $\mathrm{n}^{\circ} 81$, 1997. p. 33-39.

Su K. Hoteit N. - « Mechanical characteri. zation of an underground laboratory site in granite 1 . Proceedings of the 9th Int. Conf. on Computer Methods and Advances in Geomechanics, Wuhan. China, 2-7 November 1997.

Su K. - a Étude expérimentale de l'endomagement et de la fracturation du gra. nite de la Vienne x. Rapport pour I'ANDRA BRPOG3S 97-004, 1997.

Witherspoon P.A. Wang J.S.Y. Iwai K. Gale J.E. - Valid of Cubic Law for Fluid Flow in a Deformable Rock Fracture 1. Water Resources Research vol. $16, \mathrm{n}^{\circ} 6$. 1980, p. 1016-1024.

Zhao J., Brown E.T. - "Hydro-thermomechanical properties of joints in the Carnmenellis granite x. Publication Quarterly Journ. of Eng.geology, 25, 1992, p. 279.290. 